Check for updates

Cite this: RSC Adv., 2017, 7, 20480

\title{
Protective effects of a cocktail of lactic acid bacteria on microcystin-LR-induced hepatotoxicity and oxidative damage in BALB/c mice
}

\author{
Jichun Zhao, $\dagger^{\mathrm{a}}$ Fengwei Tian, $\dagger^{\mathrm{ac}}$ Qixiao Zhai, ${ }^{\text {ac }}$ Ruipeng $\mathrm{Yu},{ }^{\mathrm{a}}$ Hao Zhang, ${ }^{\text {ad }}$ \\ Zhennan Gu (D) and Wei Chen (DD *abd
}

\begin{abstract}
The aim of this study was to investigate the effects of mixed lactic acid bacteria (LAB) against microcystinLR-exposed hepatotoxicity and oxidative stress in BALB/c mice. $20 \mathrm{LAB}$ strains were randomly divided into three groups and the effects against microcystin-LR (MC-LR) toxicity investigated in vivo. The antioxidative abilities of the three $L A B$ groups were also determined. The results showed that a cocktail of LAB 3 (CLAB3), including Lactobacillus johnsonii ATCC 33200, L. rhamnosus GG, Bifidobacterium adolescentis 1.1290 and B. bifidum CCFM 16, was able to significantly reverse the increased levels of serum ALT and AST, and liver tissue lesions by MC-LR. The alterations of hepatic malondialdehyde (MDA), superoxide dismutase (SOD) and catalase (CAT) activities in the CLAB3 were improved compared to the MC-LR group alone. Moreover, the free radical scavenging and reducing abilities of CLAB3 were higher than other groups. CLAB3 can significantly alleviate MC-LR-induced hepatic damage and improve its oxidative stress. Moreover, the protective effects of the CLAB3 groups are related to its outstanding antioxidative abilities in vitro. $\mathrm{LAB}$ can be a promising dietary strategy to prevent cyanobacteria contamination toxicity.
\end{abstract}

Received 14th March 2017 Accepted 31st March 2017 DOI: 10.1039/c7ra03035e rsc.li/rsc-advances inhibiting specific protein phosphatases and depleting antioxidant substances. In addition, studies have reported other harmful effects of MC-LR, including reproductive toxicity in female and male mice, liver tumor progression, and intestinal disease. ${ }^{4-6}$ Oxidative damage is another important mechanism of MC-LR-exposed toxicities. ${ }^{7}$ MC-LR exposure can cause a decrease of superoxide dismutase (SOD), catalase (CAT) and glutathione (GSH) in the liver and significant increase in the malondialdehyde (MDA) level in mice model, suggesting that MC-LR-induced pro-oxidants caused antioxidant system damage. ${ }^{8,9}$

The balance between antioxidant and oxidant common dependent on endogenous protective substances and enzyme. ${ }^{\mathbf{1 0}}$ Once pro-oxidants overwhelm antioxidant system, dietary antioxidant supplements can help to keep the antioxidant system. Some researchers have focused on prevention or methods to decrease MC-LR-induced toxicity by physiological antioxidants, due to its current poor therapeutic chances. Several studies have revealed that phytochemical substances, such as green tea polyphenols, sulforaphane, and flavonoids, are effective in prevention of MC-LR-induced hepatotoxicity and oxidative damage. ${ }^{\mathbf{8 , 1 1 , 1 2}}$

Many lactic acid bacteria (LAB) strains are reported to have free radical scavenging ability, reducing ability, metal ion chelating ability and inhibiting pro-oxidative enzyme. ${ }^{\mathbf{1 3 , 1 4}}$ Moreover, some selected lactobacillus played an important role in alleviating oxidative stress in vivo..$^{15,16}$ On the other hand, numerous studies have found that some Lactobacillus and Bifidobacterium, including Lactobacillus rhamnosus, Bifidobacterium breve, and L. plantarum, can remove MC-LR or decrease its 
toxicity. ${ }^{17-19}$ However, almost all of these studies were conducted in vitro, and to our knowledge, no studies on the effects of $\mathrm{LAB}$ against MC-LR in vivo have been reported. Therefore, it is necessary to investigate the effect of LAB on MC-LR-induced toxicity in vivo. However, the effect of single LAB on MC-LRexposure mice was not significant in our previous studies, although they had good adsorption capacity of MC-LR and strong antioxidative ability in vitro (data not published). VSL\#3, a cocktail of eight different LAB strains, significantly improved MDA level and 4-hydroxynonenal in non-alcoholic fatty liver disease (NAFLD) patients. ${ }^{20}$ Alisi et al. also found that VSL\#3 supplement alleviated NAFLD in obese children, which could be related to glucagon-like peptide 1 increase. ${ }^{21}$ VSL\#3 had a stronger ability to colonize the large bowel compared to that of bifidobacteria in this cocktail. ${ }^{22}$ We therefore supposed that mixed LAB had an advantage over individual strains because of their interaction and synergism.

In this study, we selected a batch of LAB from the list of eatable bacteria released by National Health and Family Planning Commission of the People's Republic of China (PRC). We aimed to evaluate the protective effects of three different groups of LAB strains against MC-LR-induced oxidative stress and hepatotoxicity in $\mathrm{BALB} / \mathrm{c}$ mice and to investigate the differences in protective ability among the three mixed LAB groups. Furthermore, the preliminary protective mechanisms of mixed LAB groups were also examined and discussed.

\section{Materials and methods}

\section{Chemicals and kits}

MC-LR (purity $>98 \%$, by high-performance liquid chromatography) was purchased from Zen-U Biotechnology (Taiwan, China). MC-LR was firstly dissolved in the minimal amount of methanol (final concentration $0.1 \%, \mathrm{w} / \mathrm{v}$ ) and diluted to $100 \mu \mathrm{g}$ $\mathrm{mL}^{-1}$ stock solution with Mill-Q water and stored at $-20{ }^{\circ} \mathrm{C}$ until use. Methanol, ethanol, potassium ferricyanide, $\mathrm{FeSO}_{4}$, $\mathrm{H}_{2} \mathrm{O}_{2}$, trichloroacetic acid and ferrichloride were obtained from Sinopharm Chemical Reagent Company (Shanghai, China). 2,2diphenyl-1-picrylhydrazyl (DPPH), 1,10-phenanthroline, $\mathrm{N}$-acetylcysteine (NAC) and cysteine were purchased from SigmaAldrich (St. Louis, MO, USA).

Kits used to measure the levels of aspartate transaminase (AST), alanine transaminase (ALT), superoxide dismutase (SOD), catalase (CAT), malondialdehyde (MDA), total cholesterol (TC) and triglycerides (TG) were purchased from Nanjing Jiancheng Institute of Biotechnology.

\section{Bacterial strains and culture condition}

20 strains of LAB were obtained from different culture collections. The bacterial collections are from the list of eatable bacteria released by National Health and Family Planning Commission of the PRC (http://www.moh.gov.cn/mohbgt/ s10787/201004/47133.shtml). These strains were randomly separated into three groups of 11,5 , and 4 strains according to the random number generation function of SPSS version 16.0. The strain suppliers and randomized groups are shown in
Table 1 Origin and randomized groups of lactic acid bacterial strains

\begin{tabular}{llll}
\hline No. & Strains & Origin $^{a}$ & Groups \\
\hline 1 & L. acidophilus CCFM 137 & 1 & 1 \\
2 & L. casei Zhang CCFM 5 & 1 & 2 \\
3 & L. casei Zhang CCFM 9 & 1 & 1 \\
4 & L. fermentum CCFM 620 & 1 & 1 \\
5 & L. fermentum (CCFM 421 & 1 & 1 \\
6 & L. gasseri CCFM 15 & 1 & 1 \\
7 & L. helveticus CCFM 6 & 1 & 1 \\
8 & L. johnsonii ATCC 33200 & 2 & 3 \\
9 & L. plantarum CCFM 8610 & 1 & 1 \\
10 & L. plantarum CCFM 8661 & 1 & 1 \\
11 & L. reuteri CICC 6226 & 3 & 1 \\
12 & L. rhamnosus GG & 2 & 3 \\
13 & L. rhamnosus CCFM 237 & 1 & 2 \\
14 & L. salivarius Z5 CCFM 8605 & 1 & 2 \\
15 & B. adolescentis 1.2190 & 4 & 3 \\
16 & B. animalis 1.3003 & 4 & 1 \\
17 & B. animalis 1.1852 & 4 & 2 \\
18 & B. bifidum CCFM 16 & 1 & 3 \\
19 & B. breve 1.3001 & 4 & 2 \\
20 & B. breve 1.2213 & 4 & 1
\end{tabular}

${ }^{a}$ (1) The Culture Collections of Food Microbiology (CCFM), Jiangnan University (Wuxi, China); (2) American type culture collection (ATCC); (3) China Center of Industrial Culture Collection (CICC) (Beijing, China); (4) China General Microbiological Culture Collection Center (CGMCC) (Beijing, China).

Table 1. These frozen strains were inoculated in de Man, Rogosa, and Sharpe (MRS) medium and transferred three times at $37{ }^{\circ} \mathrm{C}$ for $16 \mathrm{~h}$ prior to experiments, respectively. For Bifidobacterium, L-cysteine hydrochloride was added to MRS medium (final concentration $0.1 \%, \mathrm{w} / \mathrm{v}$ ).

After cultured at $37{ }^{\circ} \mathrm{C}$ for $16 \mathrm{~h}$, these cultured strains at exponential phase were harvested and centrifuged at $3000 \mathrm{~g}$ for $10 \mathrm{~min}$ and washed three times with phosphate-buffered saline solution (PBS; $0.01 \mathrm{M}, \mathrm{pH}$ 7.4) prior to their administration in the animal trials. The cell pellets of the twenty strains were mixed into three groups according to the randomization (Table 1), lyophilized with skimmed milk as a protecting agent, and stored below $4{ }^{\circ} \mathrm{C}$. The number of bacteria in the lyophilized preparations was determined by plate colony-counting before the animal trials to calculate the administrated volume and ensure $10^{9}$ colony-forming units (CFU) for each mouse. For determination of antioxidative abilities, the cell pellets of the three groups were resuspended in PBS after washing three times with PBS, and the

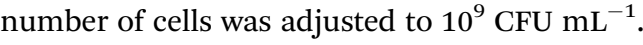

\section{Animal studies}

All protocols for animal trials were approved by the Ethics Committee of Jiangnan University (JN no. 20141010-0127-39) and performed according to the European Community guidelines (directive 2010/63/EU). All efforts were conducted to minimize suffering. Male BALB/c mice (8 weeks old; SPF; $25 \pm 2$ g) were obtained from Shanghai Laboratory Animal Center (Shanghai, China). These mice were placed in a standard animal room that was controlled at temperature $\left(24^{\circ} \mathrm{C}\right)$ and humidity $(50 \%)$, and maintained with a 12 hour light/12 hour dark cycle. They were fed 
commercial rodent chow, and free access to water. After one week of acclimatization, 54 mice were randomly separated into six groups of nine. 20 strains of LAB were randomly divided into three groups for animal studies in order to evaluate different cocktails in improving microcystin-LR toxicity.

As shown in Table 2, the experimental processes comprised three steps. The mice of the protective groups (III, IV, and V) received oral administration of cocktails of $\mathrm{LAB}$ by gavage for 14 days before injection of MC-LR; meanwhile, the mice in groups I, II and VI received gavage of skimmed milk only. During the following week, the toxin-treated mice (groups III, IV and V) received intraperitoneal injections of MC-LR at $45 \mu \mathrm{g} \mathrm{kg}{ }^{-1}$ b.w. per day 1 hour after oral administration of mixed LAB, while the mice of groups VI were treated by gavage with NAC administration instead of LAB. The group I were treated with skimmed milk and saline solution. The cocktails of LAB (CLAB) were given for two weeks after administration of MC-LR ended.

During the 5 week period, body weight was measured weekly. All mice were sacrificed after the 5 week experiments. The body weight of every mouse was weighted before sacrifice. Blood samples were collected, and centrifuged at $2000 \mathrm{~g}$ for $10 \mathrm{~min}$ to obtain serum for determining of serum ALT, AST, TC, and TG levels. The livers were removed, washed in cold saline solution for three times, dried by filter paper and weighted. Liver body weight index was calculated as liver weight in proportion to body weight. Some amount of liver were homogenized for determination of antioxidant enzyme activity. Other parts of the specimens from the liver were removed and fixed in $10 \%$ formaldehyde solution (PBS pH 7.4, v/v) for histopathological studies.

\section{Determination of serum transaminase, TC, and TG levels}

The levels of serum ALT, AST, TC, and TG were measured according to the manufacturer's recommendation with corresponding kits purchased from the Nanjing Jiancheng Bioengineering Institute.

\section{Determination of antioxidant enzyme activities and MDA levels in liver}

The livers were rinsed and homogenized in ice-cold PBS to obtain a $10 \%$ homogenate $(\mathrm{w} / \mathrm{v})$. The homogenates were centrifuged at $3000 \mathrm{~g}$ and $4{ }^{\circ} \mathrm{C}$ for 10 minutes, and the supernatant was separated. The liver homogenate supernatant was used to determine antioxidant enzyme SOD and CAT using corresponding commercial assay kits. As an indicator of lipid peroxidation, MDA was detected by determining the concentration of the reaction product of MDA and thiobarbituric acid in fluorescence at $532 \mathrm{~nm}$ with a trace MDA detection kit.

\section{Histopathological studies}

Specimens were collected from the same regions of the liver from each mouse and fixed in $10 \%$ formaldehyde overnight at room temperature. The tissues were embedded in paraffin blocks and cut at $4 \mu \mathrm{m}$ thicknesses. The paraffin-embedded tissue sections were stained with hematoxylin and eosin for microscopic examination.

\section{Measurement of DPPH radical scavenging ability}

The scavenging of DPPH by three groups of CLAB was determined with a modification of the method utilized. ${ }^{23}$ One-

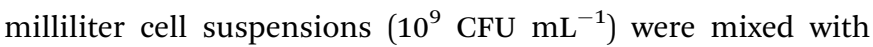
equivalent volume of fresh DPPH solution $\left(0.2 \mathrm{mmol} \mathrm{L}^{-1}\right.$ in ethanol), briefly blended, and allowed to react for 30 minutes in the dark. Blank controls consist of equal parts of PBS and ethanol. The scavenged DPPH radical was then determined by measuring the decrease in absorbance at $517 \mathrm{~nm}$. The scavenging ability was defined as follows:

$$
\text { Scavenging rate }(\%)=\left[1-A_{517(\text { sample) }} / A_{517 \text { (blank) }}\right] \times 100 \%
$$

\section{Measurement of hydroxyl radical scavenging ability}

The method of hydroxyl radical scavenging ability of three groups of LAB strains was determined as previously described. ${ }^{24}$ The hydroxyl radical scavenging ability was defined as follows:

$$
\begin{aligned}
\text { Scavenging rate }(\%)= & {\left[A_{536(\text { sample })}-A_{536(\text { blank })}\right] / } \\
& {\left[A_{536(\text { control })}-A_{536(\text { blank })}\right] \times 100 \% }
\end{aligned}
$$

Table 2 Animal experimental protocol

Treatment (days)

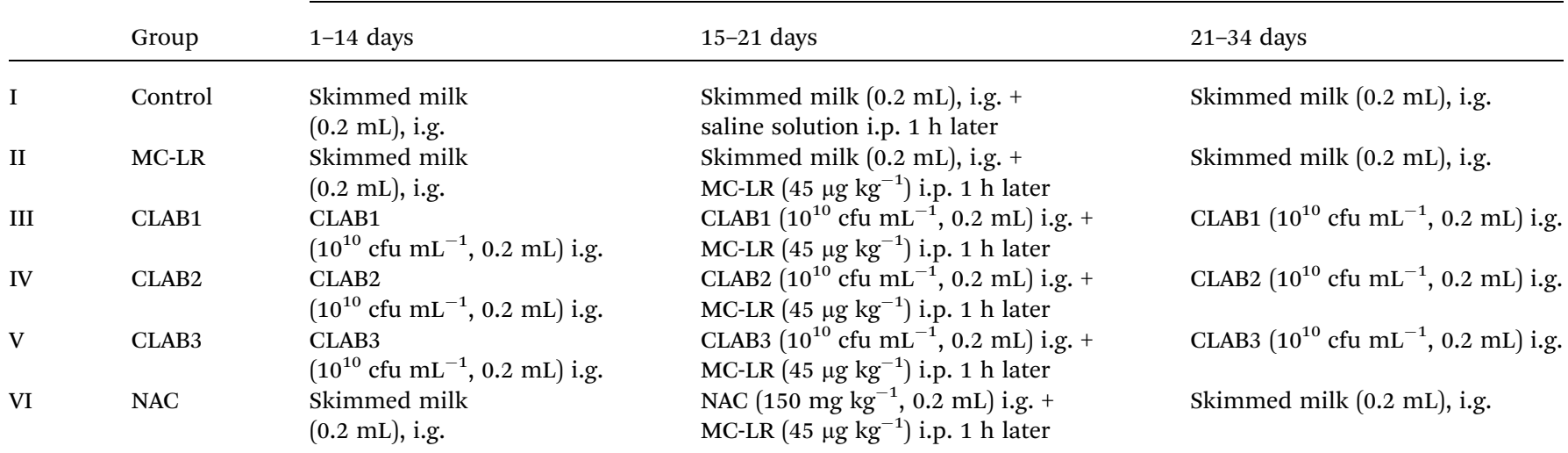




\section{Measurement of reducing activity}

The reducing activity was evaluated with a method used by. ${ }^{\mathbf{1 3}}$ After the reaction mixture was centrifuged at $3000 \mathrm{~g}$ for 5 minutes, a $1 \mathrm{~mL}$ portion of the supernatant was then added to 1 $\mathrm{mL}$ of ferrichloride $(0.1 \%, \mathrm{w} / \mathrm{v})$. The reducing activity was expressed with equivalent cysteine. Specifically, reducing activity of a series of known concentrations of L-cysteine was evaluated as the above method to build standard curve, with $\mathrm{OD}_{700}$ on the $X$ axis and the concentrations of $\mathrm{L}_{\text {-cysteine on the } Y}$ axis. The reducing activity of mixed LAB was calculated on the base of L-cysteine standard curve.

\section{Statistical analysis}

Results were expressed as the mean \pm SEM. The statistical differences between groups were analyzed by one-way analysis of variance (ANOVA) followed by Tukey's post hoc test. A $p$ value of less than 0.05 was considered to indicate statistical significance. Statistical analyses were performed using SPSS 16.0 software.

\section{Results}

\section{Body weight and liver body weight index}

All mice showed an obvious weight gain during the first 2 weeks. The weights of the MC-LR-treated mice began to decrease after intraperitoneal administration of $45 \mu \mathrm{g} \mathrm{kg} \mathrm{kg}^{-1}$ b.w. MC-LR, whereas the control mice continued to gain weight. The administration cocktails of CLAB3 obviously diminished the decrease in weight in these mice. For other intervention group and NAC group, the weights of mice recovered more quickly after quitting MC-LR administration. In the end, the weights of all groups reached nearly the same level except the only MC-LR treated group (Fig. 1). The liver body weight index of MC-LR group was bigger than others (Table 3 )

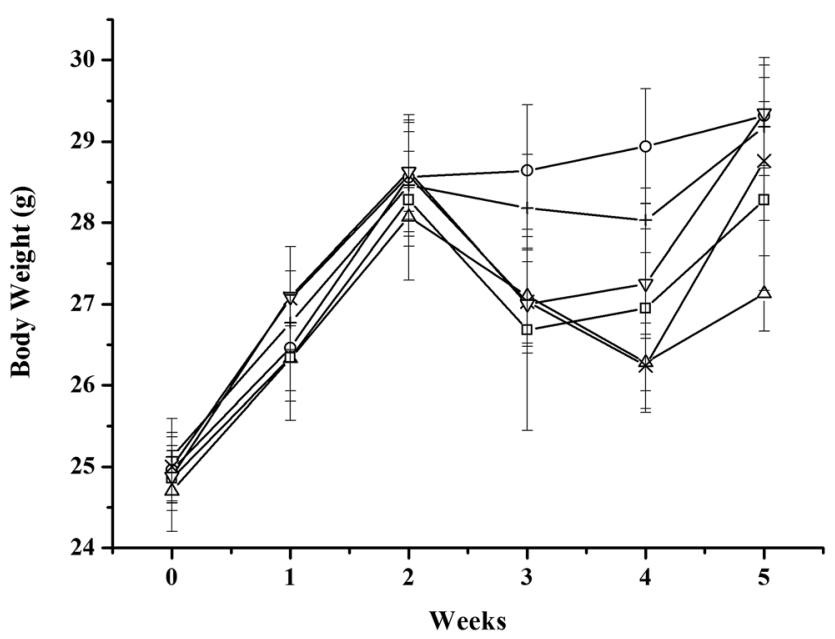

Fig. 1 Effects of mixed $L A B$ administration on the body weights of mice (C) control, $(\triangle) M C-L R$, $(\square) M C-L R+C L A B 1,(X) M C-L R+C L A B 2$, $(+)$ MC-LR + CLAB3, $(\nabla)$ MC-LR + NAC. Data are mean \pm SEM of 9 mice per group.
Table 3 Liver body weight index ${ }^{a}$

\begin{tabular}{llll}
\hline Group & Final weight & Liver weight & Liver body weight index \\
\hline Control & $29.32 \pm 0.34^{\mathrm{b}}$ & $1.42 \pm 0.01$ & $4.85 \pm 0.02^{\mathrm{a}}$ \\
MC-LR & $27.13 \pm 0.47^{\mathrm{a}}$ & $1.48 \pm 0.02$ & $5.46 \pm 0.14^{\mathrm{b}}$ \\
CLAB1 & $28.28 \pm 0.41^{\mathrm{a}, \mathrm{b}}$ & $1.40 \pm 0.01$ & $4.97 \pm 0.05^{\mathrm{a}}$ \\
CLAB2 & $28.76 \pm 0.46^{\mathrm{a}, \mathrm{b}}$ & $1.42 \pm 0.01$ & $4.89 \pm 0.05^{\mathrm{a}}$ \\
CLAB3 & $29.18 \pm 0.32^{\mathrm{b}}$ & $1.43 \pm 0.01$ & $4.88 \pm 0.02^{\mathrm{a}}$ \\
NAC & $29.35 \pm 0.32^{\mathrm{b}}$ & $1.42 \pm 0.01$ & $4.86 \pm 0.02^{\mathrm{a}}$
\end{tabular}

${ }^{a}$ The superscript letters a and $\mathrm{b}$ indicate statistically differences $(p<$ $0.05)$ between the groups in each row.

\section{Serum transaminase, TC, and TG levels}

As shown in Fig. 2, compared with the control group, MC-LR exposure caused significant increases in serum ALT and AST activity $(p<0.05)$, indicating severe hepatotoxicity. Although three different groups underwent administration of LAB before and after MC-LR exposure for 2 weeks, respectively, only the CLAB3 group successfully reversed the elevation of ALT $(-41 \%)$ and AST $(-36 \%)$ activities and alleviated liver damage. NAC administration caused a slight decrease in the ALT and AST levels compared with the MC-LR-exposed mice. A slight but nonsignificant increase was seen in the TC level in the MC-LR group. The TG level was significantly increased $(p<0.05)$. It seems that MC-LR treatment also affected the metabolism of lipid in the liver. However, in mixed LAB-treated and $N$ acetylcysteine-treated groups, the TG levels had recovered to normal.

\section{SOD, CAT, and MDA in the liver}

In the liver, MC-LR administration resulted in a significant decrease of SOD activity and an increase in the CAT level ( $p<$ 0.05) compared with the control group (Fig. 3). The CLAB treatments had a positive effect on the alteration of CAT and SOD activity, especially CLAB3, which reversed the alterations to normal levels. The antioxidant activities of CLAB3 seemed to suppress MC-LR-induced oxidative liver damage. The positive NAC also recovered their levels of SOD and CAT. In addition, a significant increase in the MDA content was observed in the MC-LR-treated group, which indicates that MC-LR caused lipid peroxidation. However, neither CLAB nor NAC treatment had any effect on the content of MDA.

\section{Liver histopathological studies}

On histologic examination, in the control group, the livers showed normal histology. A central vein was localized in the middle area of the liver lobules, with hepatic plates extending from the central vein toward the portal regions (Fig. 4a). Obviously, MC-LR exposure caused injury to liver, including loss of intact liver plates, shrinking of the cytoplasm, condensed chromatin, inflammatory cellular infiltration, and necrosis of hepatocytes (Fig. 4b). Histologic alterations were alleviated in the CLAB treatment groups. Pretreatment with CLAB3 significantly alleviated hepatic injury such as loss of 
(a)

intact hepatic plates and necrosis of hepatocytes, whereas the histological changes in the other groups were not so visible (Fig. 4). However, inflammatory cellular infiltration was still seen in mixed LAB group. It seemed that the specially mixed LAB are able to prevent hepatic damage by MC-LR. Moreover, pretreatment with NAC also preserved normal hepatic tissue structures.

\section{Antioxidative activities of mixed LAB in vitro}

The DPPH radical scavenging rate of the CLAB3 group was significantly greater than those of the CLAB1 and CLAB2 groups (Table 4). The hydroxyl radical is the most reactive oxygen radical, and thus the ability to scavenge hydroxyl radicals is of importance. The hydroxyl radical scavenging of the CLAB2 group was lower than that of the other mixed LAB groups. In addition, the reducing ability of the CLAB3 group also was greater than that of CLAB1 and CLAB2. In conclusion, the antioxidative activities of the CLAB3 group were better than those of the other two mixed LAB groups. (b)

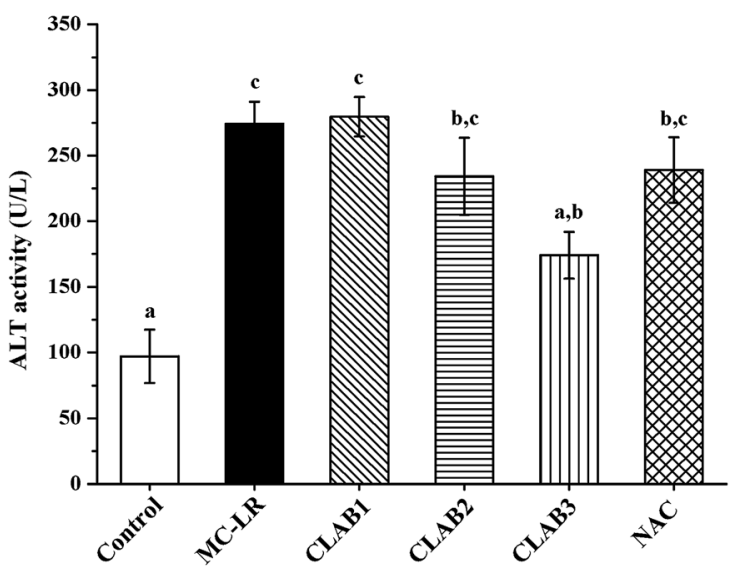

(d)

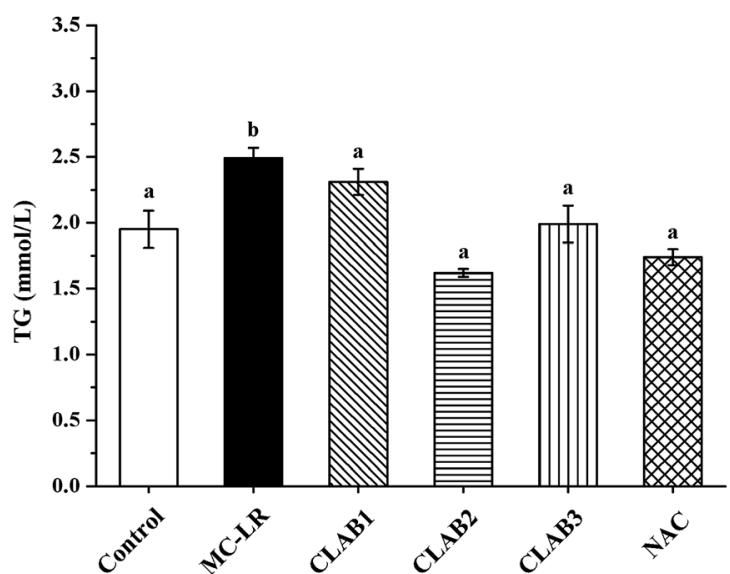

Fig. 2 Effects of a cocktail of LAB on the serum enzyme and biochemical indicates: (a) AST activity; (b) ALT activity; (c) TC contents; (d) TG contents. Data are presented as the mean \pm SEM per group. Significant differences $(p<0.05)$ are shown with different superscripts.

\section{Discussion}

Cyanobacteria toxin-induced illness and deaths have been reported worldwide in both human beings and animals. ${ }^{1,2}$ As one of the most toxic and common cyanobacteria toxins, MC-LR is reported to cause liver damage, gastrointestinal apoptosis, reproductive toxicity, kidney injuries, and immunosuppression in rodents. ${ }^{4,5,25-27}$ The strategic solutions for reducing MC-LR exposure mainly include removal, degradation and decrease intake without regard to environmental governance. Several studies have suggested that MC-LR may be degraded by LAB; in particular, the cell-envelope proteinases involved in the degradation of MC-LR were identified in Lactobacillus and Bifidobacterium strains. ${ }^{28}$ In contrast, the removal of microcystins by $\mathrm{LAB}$ in vitro is supposed to be a function of physical adsorption rather than metabolism. ${ }^{29-31}$ The mechanisms of LAB effect on MC-LR in vitro is still controversial. However, only limited studies have reported the prevention against MC-LR-toxicity in vivo. In this study, MC-LR was administered via intraperitoneal 

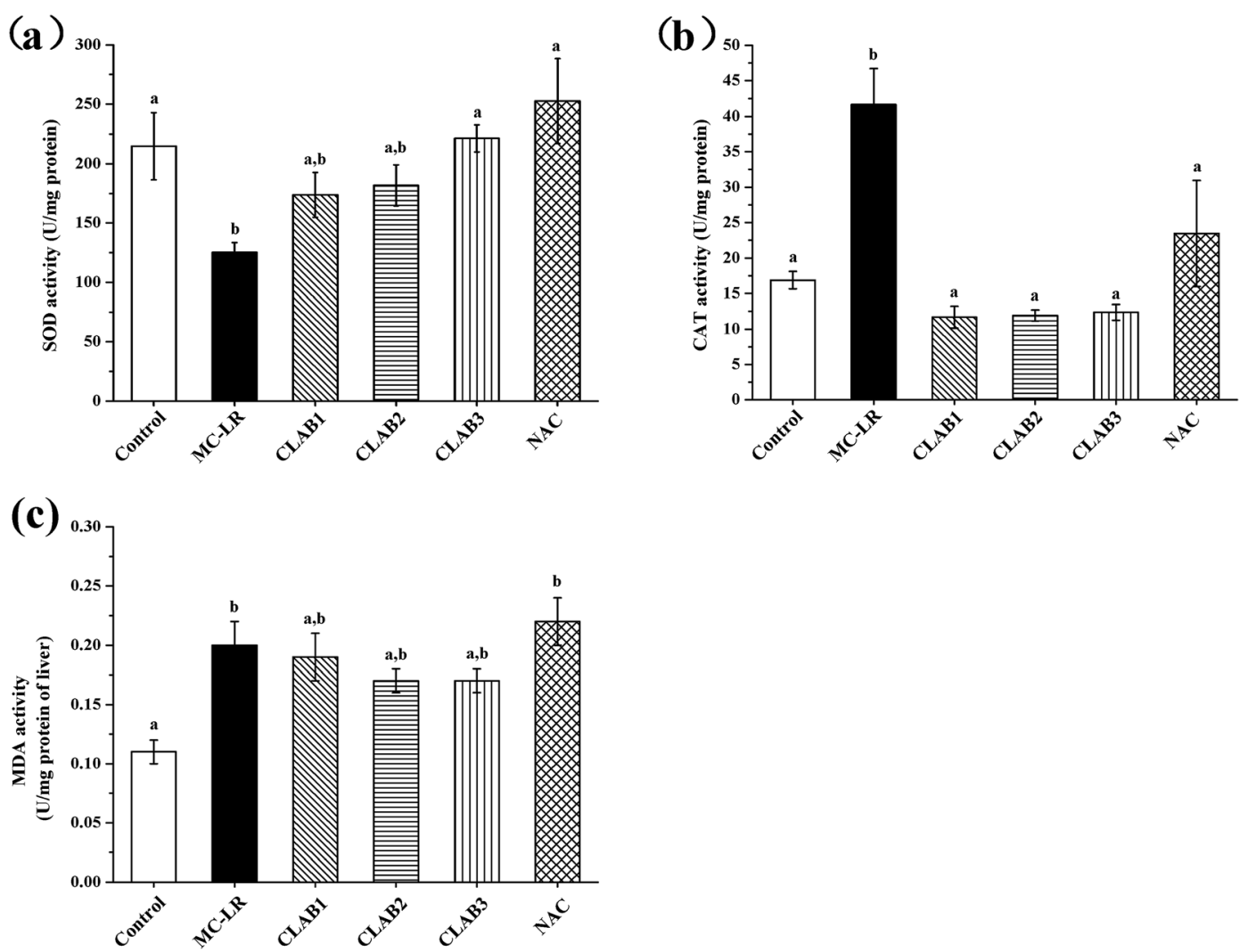

Fig. 3 Effects of a cocktail of LAB on the antioxidant enzymes in livers of MC-LR-exposed mice: (a) SOD activity; (b) CAT activity; (c) MDA contents. Data are presented as the mean \pm SEM per group. Significant differences $(p<0.05)$ are shown with different superscripts.

injection rather than by an intragastric route to avoid contact between the MC-LR and the mixed LAB to investigate the effects of LAB on MC-LR-induced toxicity in vivo. Our study may be the first to confirm the protective effects of LAB against MC-LRexposed hepatotoxicity and oxidative stress in an animal model, characterized by reducing lipid peroxidation, alleviating liver lesion, and protecting antioxidant enzymes system.

Liver is reported to be the specific affected organ by MC-LR due to its uptake through bile acid carrier system in hepatic cells. $^{32}$ The toxin inhibits protein serine/threonine phosphatases 1 and $2 \mathrm{~A}$ through its methy-dehydroalanine binding Cys $^{273}$ of the phosphatases, causing excessive phosphorylation of proteins and subsequently hepatocytes damage such as loss of cell shape, DNA lesion and hepatic hemorrhage. ${ }^{3-35}$ In the presenting study, liver damage was caused by a sublethal dose of MC-LR, with an increase in serum ALT and AST, which was significantly prevented by oral administration of CLAB3. The histologic results also demonstrated that CLAB3 treatment preserved more normal tissue structures with less cytoplasmic shrinkage, few hepatic necrosis and normal liver weight, compared with the only MC-LR group. Administration of LAB with high antioxidative activity enhances the level of glutathione peroxidase, which is crucial for the detoxification of MCLR by the glutathione pathway. ${ }^{36,37}$ However, whether CLAB3 have the same hepatoprotective pathway needs to further investigate. CLAB3 administration also diminished the decrease in weight of mice seen during and after this toxin treatment, which may be related to improvement of the inhibition of nutrient absorption by MC-LR. ${ }^{38}$ Our study provides biological evidence that mixed $\mathrm{LAB}$ can protect mice from hepatotoxicity by MC-LR. Furthermore, the protective effect of CLAB3 on the liver was even better than that of the positive control NAC. Compared to NAC group, CLAB3 group had the lower levels of serum ALT and AST, which were regarded as indices of liver function, although there was no significant difference in stained liver section between CLAB3 and NAC group.

Moreover, several studies have suggested that oxidative stress plays an important role in the pathogenic mechanism of MC-LR-exposed toxicity, ${ }^{7}$ which may be caused by an increase in reactive oxygen species and a decrease in antioxidant levels. Reactive oxygen species, such as ${ }^{\circ} \mathrm{OH}, \mathrm{O}_{2}{ }^{-}{ }^{-}$, and ROO', come mainly from the mitochondrial transport chain and enzyme during oxidative phosphorylation. Once the balance between reactive oxygen species and an antioxidative system is broken, oxidative stress can induce severe damage to cell structures by causing destruction of lipids, proteins, and DNA. In this study, CLAB3 induced recovery of the antioxidative levels by reducing the CAT activities and MDA levels and increasing the levels of SOD in the liver. The results suggest that CLAB3 can significantly prevent the oxidative damage caused by MC-LR treatment. Similarly, many Lactobacillus and Bifidobacteria are reported to have antioxidative abilities including reducing lipid peroxidation, enhancing antioxidative enzyme and improving 

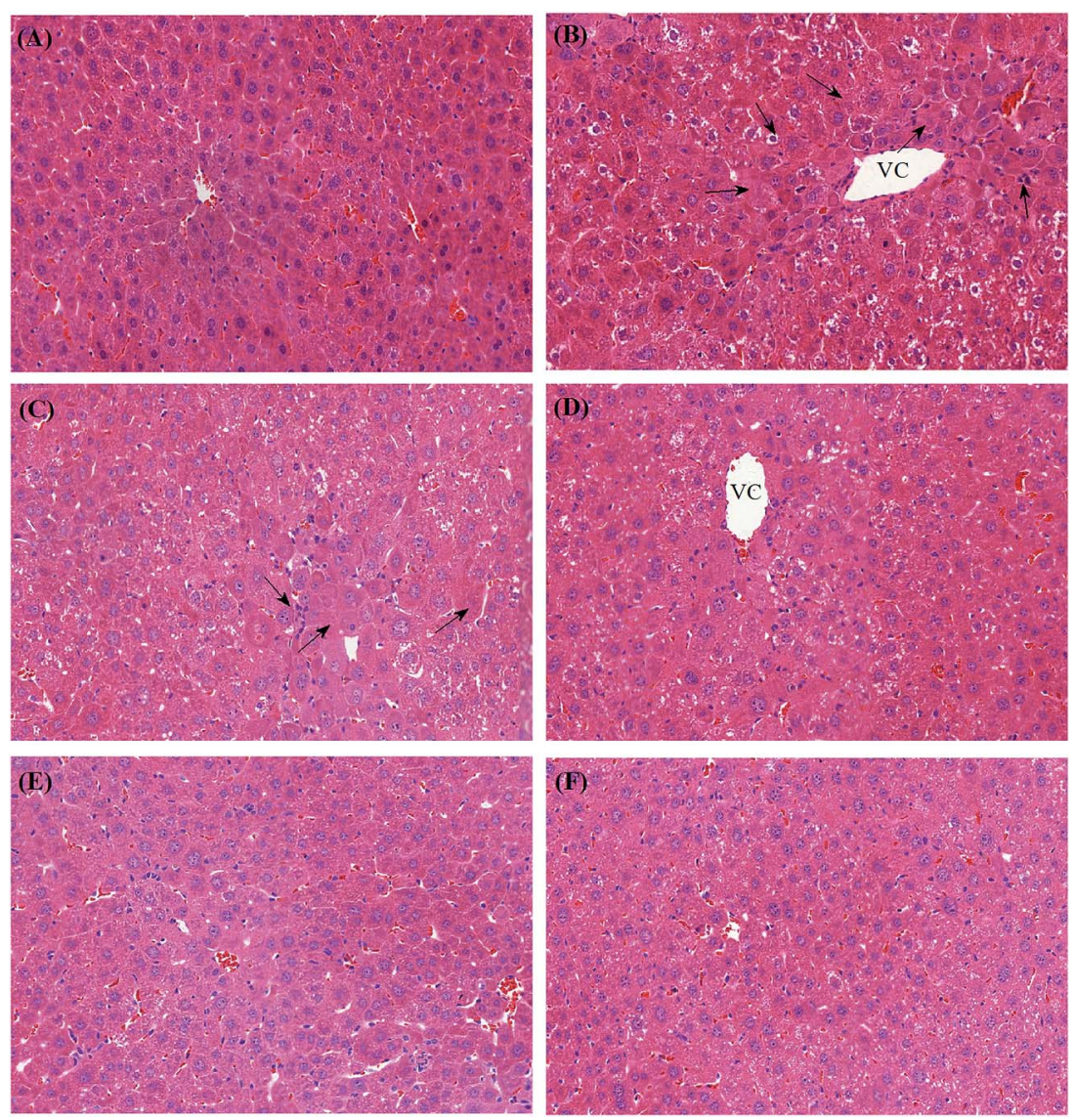

Fig. 4 Effect of mixed LAB on histological changes of liver in mice administered MC-LR: (a) liver of control, $\times 200$; (b) liver of mice treated with MC-LR, arrows indicating loss of intact liver plates, shrinking of the cytoplasm, condensed chromatin, inflammatory cellular infiltration and necrosis, $\times 200$; (c-e) and (f), liver of MC-LR-exposed mice pretreated with CLAB1, CLAB2, CLAB3 and N-acetylcysteine, respectively, $\times 200$.

Table 4 Determination of antioxidative activities of three groups of mixed LAB strains ${ }^{a}$

\begin{tabular}{llll}
\hline Group & $\begin{array}{l}\text { DPPH radicals } \\
\text { scavenging }(\%)\end{array}$ & $\begin{array}{l}\text { Hydroxyl radicals } \\
\text { scavenging }(\%)\end{array}$ & $\begin{array}{l}\text { Reducing ability } \\
(\mu \mathrm{mol})\end{array}$ \\
\hline CLAB1 & $25.92 \pm 0.87^{\mathrm{a}}$ & $78.76 \pm 1.56^{\mathrm{b}}$ & $30.04 \pm 2.00^{\mathrm{a}}$ \\
CLAB2 & $26.40 \pm 1.25^{\mathrm{a}}$ & $66.42 \pm 1.17^{\mathrm{a}}$ & $52.81 \pm 1.63^{\mathrm{b}}$ \\
CLAB3 & $32.06 \pm 0.56^{\mathrm{b}}$ & $76.73 \pm 0.98^{\mathrm{b}}$ & $75.93 \pm 1.69^{\mathrm{c}}$
\end{tabular}

${ }^{a}$ Values are mean \pm SEM for 3 times per group. The superscript letters a and $\mathrm{c}$ indicate statistically differences $(p<0.05)$ between the groups in each row. ${ }^{b}$ Reducing activity was expressed with equivalent cysteine $\left(\mu \mathrm{mol} \mathrm{L}{ }^{-1}\right)$.

lipid metabolism. ${ }^{15,16}$ Due to CLAB3 including L. johnsonii ATCC 33200 , L. rhamnosus GG, B. adolescentis 1.1290 and B. bifidum CCFM 16, it is difficult to figure out the dominante of these strains in antioxidant ability. Therefore, it is necessary to compare every single strain with CALB3 in antioxidant ability in the future research. Moreover, the alteration of TG levels also indicated that CLAB can improve lipid metabolism in the liver.

We speculate the antioxidative of probiotics may be related to reducing ability, reactive oxygen species scavenging, enzyme inhibition, and inhibition of ascorbate autoxidation..$^{13}$ Actually, the antioxidative ability of CLAB3 is greater than others, which is consistent with the results in vivo. Although the molecular mechanisms and pathways of LAB on protective effects still require further study, the antioxidative activity of LAB may be one of the most important aspects. The positive NAC was considered as one of potent chemical substances against liver injury. ${ }^{39}$ As a glutathione precursor, NAC improved MC-LRinduced oxidative stress by increasing antioxidative enzymes, which was similar to LAB. Moreover, NAC also can prevent MCLR-induced DNA damage in HepG2 cells. ${ }^{40}$ The possible mechanisms of NAC on MC-LR toxicity included decreasing ROS production, attenuating p38 mitogen-activated protein kinase activation, elevating mitochondrial membrane potential and reducing apoptosis. ${ }^{41-43}$

In conclusion, we demonstrate for the first time that a cocktail of LAB administration conferred protection against oxidative damage and hepatotoxicity induced by MC-LR in vivo. The study indicates that the antioxidant abilities of LAB may play an important role in diminishing MC-LR-induced toxicity. Moreover, the antioxidant capacity may be related to radical scavenging abilities and reducing ability. The results show that LAB may be one of potential dietary strategies against MC-LR toxicity. 


\section{Conflict of interest}

All the authors declared that they have no conflict of interest.

\section{Acknowledgements}

This work was supported by the National Science Fund for Distinguished Young Scholars No. 31125021, the Program for Changjiang Scholars and Innovative Research Team in University IRT1249, the Program of Introducing Talents of Discipline to Universities (B07029) and the Program of Collaborative innovation center of food safety and quality control in Jiangsu Province.

\section{References}

1 S. Azevedo, W. W. Carmichael, E. M. Jochimsen, K. L. Rinehart, S. Lau, G. R. Shaw and G. K. Eaglesham, Toxicology, 2002, 181, 441-446.

2 R. M. Dawson, Toxicon, 1998, 36, 953-962.

3 W. J. Fischer, S. Altheimer, V. Cattori, P. J. Meier, D. R. Dietrich and B. Hagenbuch, Toxicol. Appl. Pharmacol., 2005, 203, 257-263.

4 J. Wu, S. Shao, F. Zhou, S. Wen, F. Chen and X. Han, Environ. Toxicol. Pharmacol., 2014, 37, 1-6.

5 N. Botha, M. van de Venter, T. G. Downing, E. G. Shephard and M. M. Gehringer, Toxicon, 2004, 43, 251-254.

6 Y. Zhou, J. Yuan, J. Wu and X. Han, Toxicol. Lett., 2012, 212, 48-56.

7 W. X. Ding and C. N. Ong, FEMS Microbiol. Lett., 2003, 220, 17.

8 C. Xu, W. Q. Shu, Z. Q. Qiu, J. A. Chen, Q. Zhao and J. Cao, Environ. Toxicol. Pharmacol., 2007, 24, 140-148.

9 A. I. Prieto, A. Jos, S. Pichardo, I. Moreno and A. M. Camean, Environ. Toxicol. Chem., 2008, 27, 1152-1159.

10 R. L. Prior, X. L. Wu and K. Schaich, J. Agric. Food Chem., 2005, 53, 4290-4302.

11 R. Jayaraj, U. Deb, A. S. Bhaskar, G. B. Prasad and P. V. Rao, Environ. Toxicol., 2007, 22, 472-479.

12 X. Sun, L. Mi, J. Liu, L. Song, F. L. Chung and N. Gan, Toxicol. Appl. Pharmacol., 2011, 255, 9-17.

13 M. Y. Lin and C. L. Yen, J. Agric. Food Chem., 1999, 47, 14601466.

14 A. A. Achuthan, R. K. Duary, A. Madathil, H. Panwar, H. Kumar, V. K. Batish and S. Grover, Mol. Biol. Rep., 2012, 39, 7887-7897.

15 Y. Zhang, R. Du, L. Wang and H. Zhang, Eur. Food Res. Technol., 2010, 231, 151-158.

16 S.-w. Zhang, L. Jia-ping, B. Menghe, L. Liu and X.-b. Hu, Sci. Agric. Sin., 2010, 43, 2141-2146.

17 T. Halttunen, M. C. Collado, H. El-Nezami, J. Meriluoto and S. Salminen, Lett. Appl. Microbiol., 2008, 46, 160165.

18 S. M. K. Nybom, M. C. Collado, I. S. Surono, S. J. Salminen and J. A. O. Meriluoto, J. Agric. Food Chem., 2008, 56, 37143720 .
19 I. S. Surono, M. C. Collado, S. Salminen and J. Meriluoto, Food Chem. Toxicol., 2008, 46, 502-507.

20 C. Loguercio, A. Federico, C. Tuccillo, F. Terracciano, M. V. D'Auria, C. De Simone and C. D. Blanco, J. Clin. Gastroenterol., 2005, 39, 540-543.

21 A. Alisi, G. Bedogni, G. Baviera, V. Giorgio, E. Porro, C. Paris, P. Giammaria, L. Reali, F. Anania and V. Nobili, Aliment. Pharmacol. Ther., 2014, 39, 1276-1285.

22 B. Bianchi-Salvadori, R. Vesely, A. Ferrari, E. Canzi, C. Casiraghi and F. Brighenti, New Microbiol., 2001, 24, 2333.

23 M. Y. Lin and F. J. Chang, Dig. Dis. Sci., 2000, 45, 1617-1622. 24 S. Zhang, L. Liu, Y. Su, H. Li, Q. Sun, X. Liang and J. Lv, Afr. J. Microbiol. Res., 2011, 5, 5194-5201.

25 R. E. Guzman and P. F. Solter, Vet. Pathol., 2002, 39, 17-26. 26 L. M. Lei and L. R. Song, Diyi Junyi Daxue Xuebao, 2005, 25, 565-566.

27 P. P. Shen, S. W. Zhao, W. J. Zheng, Z. C. Hua, Q. Shi and Z. T. Liu, Toxicol. Lett., 2003, 143, 27-36.

28 S. M. Nybom, D. Dziga, J. E. Heikkila, T. P. Kull, S. J. Salminen and J. A. Meriluoto, Toxicon, 2012, 59, 171181.

29 S. Fuchs, G. Sontag, R. Stidl, V. Ehrlich, M. Kundi and S. Knasmuller, Food Chem. Toxicol., 2008, 46, 1398-1407.

30 M. Piotrowska, Toxins, 2014, 6, 2826-2839.

31 S. Hatab, T. Yue and O. Mohamad, J. Appl. Microbiol., 2012, 112, 892-899.

32 J. K. Fawell, R. E. Mitchell, D. J. Everett and R. E. Hill, Hum. Exp. Toxicol., 1999, 18, 162-167.

33 D. M. Toivola, J. E. Eriksson and D. L. Brautigan, FEBS Lett., 1994, 344, 175-180.

34 R. Nishiwakimatsushima, T. Ohta, S. Nishiwaki, M. Suganuma, K. Kohyama, T. Ishikawa, W. W. Carmichael and H. Fujiki, J. Cancer Res. Clin. Oncol., 1992, 118, 420424.

35 R. W. Mackintosh, K. N. Dalby, D. G. Campbell, P. T. W. Cohen, P. Cohen and C. Mackintosh, FEBS Lett., 1995, 371, 236-240.

36 Y. Wang, J. Z. Zhou, X. D. Xia, Y. C. Zhao and W. L. Shao, Int. Dairy J., 2016, 62, 28-34.

37 M. M. Gehringer, E. G. Shephard, T. G. Downing, C. Wiegand and B. A. Neilan, Int. J. Biochem. Cell Biol., 2004, 36, 931-941.

38 J. He, J. Chen, L. Y. Wu, G. Y. Li and P. Xie, J. Proteome Res., 2012, 11, 5934-5946.

39 S. Sahin and O. Alatas, Indian J. Gastroenterol., 2013, 32, 311315.

40 B. Zegura, T. T. Lah and M. Filipic, Mutat. Res., Genet. Toxicol. Environ. Mutagen., 2006, 611, 25-33.

41 L. Xue, J. Li, Y. Li, C. Chu, G. Xie, J. Qin, M. Yang, D. Zhuang, L. Cui, H. Zhang and X. Fu, Int. J. Clin. Exp. Med., 2015, 8, 4911-4921.

42 G. Meng, J. Liu, S. Lin, Z. Guo and L. Xu, Environ. Toxicol., 2015, 30, 366-374.

43 A. C. Bautista, C. E. Moore, Y. Lin, M. G. Cline, N. Benitah and B. Puschner, BMC Vet. Res., 2015, 11, 1-6. 